\title{
Hydrodeoxygenation of raw bio-oil towards platform chemicals over FeMoP/zeolite catalysts
}

Idoia Hita ${ }^{\mathrm{a}, \mathrm{b}}$, Tomás Cordero-Lanzac ${ }^{\mathrm{a}}$, Giuseppe Bonura ${ }^{\mathrm{c}, *}$, Catia Cannilla ${ }^{\mathrm{c}}$, José M. Arandes $^{\mathrm{a}}$, Francesco Frusteri ${ }^{\mathrm{c}}$, Javier Bilbao ${ }^{\mathrm{a}}$

${ }^{a}$ Department of Chemical Engineering, University of the Basque Country (UPV/EHU), PO Box 644-48080, Bilbao, Spain

${ }^{b}$ Multiscale Reaction Engineering, KAUST Catalysis Center (KCC), King Abdullah University of Science and Technology (KAUST), Thuwal, 23955-6900, Saudi Arabia

${ }^{c}$ CNR-ITAE, Istituto di Tecnologie Avanzate per l'Energia "Nicola Giordano", Via S. Lucia sopra Contesse, 5-98126 Messina, Italy

*Corresponding author: idoia.hita@ehu.eus; giuseppe.bonura@itae.cnr.it 


\begin{abstract}
The hydrodeoxygenation of raw bio-oil was investigated in a continuous fixed bed reactor over a series of FeMoP/zeolite catalysts, pursuing a maximized and stable production of phenolics and aromatics. After $5 \mathrm{~h}$ of reaction, the catalysts reach a pseudosteady activity state. The FeMoP/HZSM-5 catalyst yields the highest amount of carbon products $(53.5 \mathrm{wt} \%)$ with the highest selectivity towards aromatics (13.6 wt\%, dry basis) and phenolics (12.2 wt\%), due to the synergy between the weak acidity of the FeMoP and the mild acidity of the HZSM-5 zeolite, which minimizes dehydration and gasification. The MFI framework allows the sweeping of coke precursors.
\end{abstract}

Keywords: hydrodeoxygenation; bio-oil; aromatics, phenolics; deactivation; HZSM-5 zeolite 


\section{Introduction}

Biomass-derived pyrolysis oils, typically designated as bio-oils, are steadily becoming a promising source for biofuels, hydrogen, and platform chemicals within an energetic context that strongly demands for alternative energy sources and the regulation of $\mathrm{CO}_{2}$ emissions [1,2]. Bio-oils typically consist of a 15-30 wt \% of water, with a 50-65 wt \% of a plethora of organic components [3,4]. Given its high oxygen content, bio-oil presents a low heating value, a high chemical and thermal instability, a high viscosity and a corrosive nature, hence evidencing necessity for its upgrading. In this context, hydrodeoxygenation (HDO) has been addressed for both the production of biofuels and hydrocarbons [6-8], due to its high availability in existing traditional refinery schemes.

The HDO of bio-oil comprises hydrogenation, dehydration, gasification (decarbonylation/decarboxylation) and cracking of its components, together with parallel condensation of heavy bio-oil components towards coke $[9,10]$. The composition of the bifunctional catalysts reported in the literature [11] aims to achieve a balanced synergy of the capacity of the metallic and acid functions (i) a high hydrogenation capacity and (ii) a limited advance of gasification reactions and deactivation due to coke formation. Noble metals $(\mathrm{Ru}, \mathrm{Rh}, \mathrm{Pt}, \mathrm{Pd})$ are active at a relatively low temperature and usually lead to highly hydrogenated end products [1216]. Zhang et al. [17] were able to avoid coke formation over a Ru catalyst by using super/sub-critical ethanol as a solvent for the organic fraction of bio-oil, and obtaining a hydrocarbon yield of $23.2 \mathrm{wt} \%$. In this context, the proposal of mildly active and cheap HDO metallic phases, like transition metals, is encouraging due to their limited gasification activity and a milder coke formation [18-20]. Ni-based catalysts were proposed by Zhang et al. [21,22] for the hydrotreatment of raw bio-oil also supressing coke formation by the use of supercritical ethanol at mild temperature $\left(280{ }^{\circ} \mathrm{C}\right)$. Nie et al. [23] attribute the deoxygenation activity of phenolic compounds towards aromatics in Fe-based catalysts to the oxophilic character of Fe, which favours the interaction with carbonyl groups and the scission of aromatic C-O bonds. Mo-based catalysts also provide great capacity for the adsorption of electronegative oxygen and $\mathrm{C}=\mathrm{O}$ bond activation, which is assigned to the low electron density and high electrophilicity of Mo 
[24]. The increase in the HDO activity of phosphided catalysts was attributed to the presence of PO-H groups as Bronsted acidic sites which provide active hydrogen [25].

As catalyst supports, conventional acidic supports (i.e. $\gamma-\mathrm{Al}_{2} \mathrm{O}_{3}, \mathrm{SiO}_{2}-\mathrm{Al}_{2} \mathrm{O}_{3}$, activated carbons) have been used, with a more recent inclusion of zeolites, which provide a high hydrothermal stability and also tunable acidity properties, which have a crucial role for controlling catalyst selectivity in HDO mechanisms and limiting coke formation in noble metal [26] and transition metal-based catalysts [27].

A favorable synergy for achieving high selectivity towards aromatics from aryl ethers and phenol was attained by Rensel et al. [28,29] using unsupported FeMoP catalysts, also demonstrating the stability of these catalysts in a reaction medium with high water content. In a study on the structural properties of $\mathrm{Fe}_{\mathrm{x}} \mathrm{Mo}_{2-\mathrm{x}} \mathrm{P}$ catalysts, they also correlated the lower activation energy required for the cleavage of the $\mathrm{C}-\mathrm{O}$ bond in phenol with the Lewis acidity of these catalysts, which enhance the elongation of these linkages [30]. The higher selectivity towards benzene on the HDO of phenol was reported by Jain et al. [31], with the FeMoP catalyst (up to $90 \%$ ) in contrast to the $50 \%$ and $22 \%$ achieved using the RuMoP and NiMoP catalysts, respectively. It is also worth mentioning that, despite some authors having reported HDO studies of synthetic bio-oil [27], the majority of the aforementioned advances on catalyst development have been carried out in the HDO of model components, while works dealing with real bio-oils are still lagging behind.

With this background, this work delves into the HDO of a raw bio-oil on a continuous setup targeting the stable production of platform chemicals. With the goal of progressing in terms of bifunctional catalyst tuning, and based on the information available in the literature on bio-oil model component HDO, FeMoP-based catalysts supported on different zeolites (HY, HZSM-5 and HBeta) have been studied. These zeolites possess different channel-type pore structure as well as distinct amount and strength of acidic sites, which allow for analyzing the advantages of supporting the FeMoP catalyst over a zeolite, as well as the role of their properties on HDO activity, selectivity and coke deactivation. The utilization of an acid zeolite as catalyst support for the FeMoP phase pursues increasing cracking activity, as well as facilitating the attainment of a pseudosteady state with an acceptable remaining catalyst activity for the 
production of valuable chemicals for the petrochemical industry (i.e. phenol, phenolics, aromatics). A bulk FeMoP catalyst was also used as reference.

The use of a fixed bed reactor with a continuous feed of raw bio-oil presents high interest, and specially operating in experimental conditions resembling those of operating refinery hydroprocessing units. This is a sensible strategy aiming for the viability of a large-scale biomass upgrading, by using technologically developed units. This continuous fixed bed reactor also makes the study of the catalyst stability possible and also permits a careful consideration over time on stream of the fast deactivation that occurs due to coke formation. Additionally, by processing raw bio-oil, this work represents for a realistic approach for addressing the difficulties associated with catalyst deactivation in bio-oil conversion and overcomes the operational difficulties of raw biooil handling in continuous reaction setups.

\section{Experimental}

\subsection{Catalyst synthesis and characterization}

$\mathrm{FeNO}_{3} \cdot 9 \mathrm{H}_{2} \mathrm{O}$ (Sigma Aldrich, 99\%), $\left(\mathrm{NH}_{4}\right)_{6} \mathrm{Mo}_{7} \mathrm{O}_{24} \cdot 4 \mathrm{H}_{2} \mathrm{O}$ (Panreac, 99\%) and $\left(\mathrm{NH}_{4}\right)_{2} \mathrm{HPO}_{4}$ (Baker Chemicals B.V., 98\%) were added sequentially in a stirred round bottom flask to an aqueous citric acid solution $(0.4 \mathrm{M})$, in order to obtain a Fe:Mo:P molar ratio of $1: 1: 1$, while the citric acid was present in a concentration such as to ensure a molar ratio of 2:1 with respect to all metals. For the preparation of the FeMoP/zeolite catalysts, three different commercial (Alfa Aesar) zeolite samples were used in ammonium form: zeolite $\beta$, zeolite ZSM-5, and zeolite Y (see Table S1). After the metal precursors were dissolved in citric acid, the zeolite sample was impregnated by incipient wetness to get a final metal loading close to $20 \mathrm{wt} \%$. Then it was dried overnight in oven at $200{ }^{\circ} \mathrm{C}$ and calcined in a muffle at $700{ }^{\circ} \mathrm{C}$ for $5 \mathrm{~h}$. Regarding the reference bulk FeMoP catalyst, and after dissolution of the precursors, the solution was partially dried using a rotary evaporator to form a viscous yellow-green liquid prior to being transferred to a drying dish. The viscous solution was further dried and calcined under the same conditions used for the FeMoP/zeolite samples. For all the catalysts, the activation was performed by programmed temperature treatment by: heating at $100{ }^{\circ} \mathrm{C}$ for $1 \mathrm{~h}$ under Ar atmosphere $30 \mathrm{STP} \mathrm{cm}^{3} \mathrm{~min}^{-1}$ ), followed by reduction under a $\mathrm{H}_{2}$ atmosphere $\left(100 \mathrm{STP} \mathrm{cm}^{3} \mathrm{~min}^{-1}\right)$ up to $650{ }^{\circ} \mathrm{C}$ for $1 \mathrm{~h}$ (heating rate of $5{ }^{\circ} \mathrm{C} \mathrm{min}^{-1}$ ). After 
reduction, the resulting FeMoP catalysts were cooled to room temperature under a $\mathrm{H}_{2}$ atmosphere and then passivated using a $1 \% \mathrm{O}_{2} / \mathrm{He}$ mixture $\left(30 \mathrm{STP} \mathrm{cm}^{3} \mathrm{~min}^{-1}\right)$ for $1 \mathrm{~h}$.

The chemical composition of the samples was determined by inductively coupled plasma mass spectrometry (ICP-MS) in a Perkin-Elmer DRC-e. Samples were calibrated using standards prepared on a mass basis.

The textural properties of the fresh/activated catalysts were determined by $\mathrm{N}_{2}$ adsorption-desorption at $-196^{\circ} \mathrm{C}$ using a Micromeritics ASAP 2020 device. Surface area was assessed using the Brunauer-Emmett-Teller (BET) equation while pore size distribution was determined by the Barrett-Joyner-Hallenda (BJH) method. The micropore volume was calculated using the $t$-method.

The acidic features of the fresh catalysts were measured through temperature programmed desorption (TPD) of $\mathrm{NH}_{3}$. The catalysts were preliminarily reduced under a $30 \mathrm{vol} \% \mathrm{H}_{2} / \mathrm{N}_{2}$ stream (100 STP $\mathrm{cm}^{3} \mathrm{~min}^{-1}$ ) at $650{ }^{\circ} \mathrm{C}$ for $1 \mathrm{~h}$. Afterwards, the catalyst samples were saturated at $150{ }^{\circ} \mathrm{C}$ for $60 \mathrm{~min}$ in a gas mixture containing $5 \mathrm{vol} \%$ of $\mathrm{NH}_{3} / \mathrm{He}$, at a total flow rate of $25 \mathrm{STP} \mathrm{cm}^{3} \mathrm{~min}^{-1}$. Then, the samples were purged under a He atmosphere until a constant baseline was attained. TPD measurements were performed in a linear quartz reactor (length, $20 \mathrm{~cm}$; internal diameter, $4 \mathrm{~mm}$ ) in the $100-800{ }^{\circ} \mathrm{C}$ temperature range at a rate of $10{ }^{\circ} \mathrm{C} \mathrm{min}^{-1}$ using $\mathrm{He}\left(25 \mathrm{STP} \mathrm{cm}^{3} \mathrm{~min}^{-1}\right)$ as carrier flow. The evolved ammonia was registered by an on-line thermal-conductivity detector, connected to a quadrupole mass spectrometer (QMS, Thermolab, Fisons Instruments) by a heated $\left(180{ }^{\circ} \mathrm{C}\right)$ inlet capillary system (transit time $\left.<0.5 \mathrm{~s}\right)$.

Temperature-programmed oxidation (TPO) of the used catalyst samples was performed in a TA Instruments TGA Q500 IR thermobalance. The used catalysts were previously stripped under a $\mathrm{N}_{2}$ stream (100 STP $\mathrm{cm}^{3} \mathrm{~min}^{-1}$ ) at $450{ }^{\circ} \mathrm{C}$ and holding that temperature for $20 \mathrm{~min}$ in order to remove non-deactivating adsorbed components. TPO analyses were performed under a continuous air flow of $100 \mathrm{STP} \mathrm{cm}^{3} \mathrm{~min}^{-1}$ by increasing temperature up to $700{ }^{\circ} \mathrm{C}$ at a $5^{\circ} \mathrm{C} \min ^{-1}$ rate and holding that temperature for $1 \mathrm{~h}$, ensuring total combustion of the deposited coke.

\subsection{Raw bio-oil}

The raw bio-oil was produced from the pyrolysis of black poplar in a pilot plant provided with a conical spouted bed reactor (CSBR), and with a capacity of $25 \mathrm{~kg} \mathrm{~h}^{-1}$ 
[32]. This bio-oil was characterized through elemental analysis in a Leco TruSpec CHN Macro apparatus provided with an additional TruSpec $\mathrm{S}$ module, and the oxygen content was determined by difference. The water content in the feed was determined by Karl-Fischer titration in a Metrohm830 KF Titrino plus equipment. On the other hand, its chemical composition was analyzed by means of gas chromatography-mass spectrometry in a Shimadzu GC-MS QP2010 unit provided with a BPX5 column (length, $50 \mathrm{~m}$; internal diameter, $0.22 \mathrm{~mm}$ ).

Table 1 lists some of the main properties of the raw bio-oil, which presents a water content of $49.0 \mathrm{wt} \%$, as well as a noteworthy oxygen concentration of $40.7 \mathrm{wt} \%$. The High Heating Value (HHV) of the oil was of $20.6 \mathrm{MJ} \mathrm{kg}^{-1}$, as calculated from Dulong's equation [33] from the elemental composition data. A more detailed chemical composition of the volatile fraction of bio-oil was reported in a previous work [34]. The high concentrations of acetic acid (23.5 wt \%), levoglucosan (24.0 wt\%) and phenolic components $(13.0 \mathrm{wt} \%)$ evidence the highly oxygenated nature of bio-oil. Phenolic components in bio-oil comprise both alkylphenolic and alkylmethoxyphenolic classes, which are highly unstable and reactive in repolymerization reactions towards the formation of coke [35].

Table 1. Physico-chemical properties of the raw bio-oil

\begin{tabular}{lc}
\hline Water content (wt\%) & 49.0 \\
Acidity & 2.3 \\
Density $\left(\mathrm{kg} \mathrm{L}^{-1}\right)$ & 1.10 \\
Elemental composition $(w t \%)$ & \\
$\quad \mathrm{C}$ & 52.2 \\
$\mathrm{H}$ & 7.1 \\
$\mathrm{O}$ & 40.7 \\
Heating Value $\left(\mathrm{MJ} \mathrm{kg}^{-1}\right)$ & 20.6 \\
\hline
\end{tabular}

\subsection{Reaction unit, product analysis and reaction parameters}

The raw bio-oil hydroprocessing runs were carried out in a down-flow fixed bed reactor schematized in detail elsewhere [36], and operating in a trickle bed regime under the following conditions: $450{ }^{\circ} \mathrm{C}$; 65 bar; space time, $0.15 \mathrm{~g}_{\text {cat }} \mathrm{h} \mathrm{g}^{-1}$ bio-oil $; 0.05 \mathrm{~cm}^{3} \mathrm{~min}^{-1}$ biooil; $90 \mathrm{~cm}^{3} \mathrm{STP} \mathrm{min}^{-1} \mathrm{H}_{2}$; and time on stream, 0-8 h. The catalyst, diluted in SiC, was loaded in the reactor as described by Van Herk et al. [37] in order to avoid heat losses and gas bypassing. Prior to the reaction, the catalysts were pre-treated at $400{ }^{\circ} \mathrm{C}$ for $12 \mathrm{~h}$ 
under a continuous gas flow mix of $30 \mathrm{~cm}^{3} \mathrm{STP} \mathrm{min}^{-1} \mathrm{H}_{2}$ and $50 \mathrm{~cm}^{3} \mathrm{STP} \min ^{-1} \mathrm{~N}_{2}$. After exiting the reactor, the reaction products were sent to a gas/liquid separator from where they were collected and weighed every hour for mass balance calculation purposes, while gases were collected and analyzed in a Agilent 300A MicroGC, provided with 4 channels with their corresponding columns: (i) a molecular sieve to separate the permanent gases $\mathrm{H}_{2}, \mathrm{O}_{2}, \mathrm{~N}_{2}$, methane and $\mathrm{CO}$; (ii) a Porapak Q to separate $\mathrm{CO}_{2}$ and water; (iii) a $\mathrm{Al}_{2} \mathrm{O}_{3}$ to separate $\mathrm{C}_{2}-\mathrm{C}_{5}$ hydrocarbons; and (iv) a Stabilwax to separate $\mathrm{C}_{6}-\mathrm{C}_{8}$ hydrocarbons, methanol and BTX aromatics.

Liquid reaction products consisted of an aqueous and an organic phase which were separated by decantation for their individual analysis. The composition of the organic product phase was assessed through two-dimensional Gas Chromatography (GCxGC) coupled with Mass Spectrometry (MS) in an Agilent 7890A apparatus connected online with an Agilent 5975C series MS. The GCxGC is provided with two columns of different polarities connected through a flow modulator, being the first column a nonpolar DB-5MS (length, $30 \mathrm{~m}$; internal diameter, $0.25 \mathrm{~mm}$ ), while the second one was a polar TRB-50 HT (length, $5 \mathrm{~m}$; internal diameter, $0.25 \mathrm{~mm}$ ). On the other hand, the aqueous phase was analyzed using the previously described Shimadzu GC-MS QP2010. The water content in the aqueous product phase was quantified by Karl-Fischer titration equally to the bio-oil feed (see Section 2.2).

The liquid carbon product (C-prod) and water yields were defined as specified in Eq. 1 on a bio-oil basis (including water), while the gas+coke yield was calculated by difference.

$\mathrm{Y}_{\mathrm{i} \text {,wet basis }}=\frac{\mathrm{F}_{\mathrm{i}}}{\mathrm{F}_{\text {bio-oil }}} 100$

where $F_{i}$ and $F_{\text {bio-oil }}$ are hourly mass flows of each product fractions and the bio-oil, respectively.

The carbon products have been grouped in lumps, as follows: oxygenates (comprising methanol, acetone and acetic acid), alkanes, ketones, phenol, phenolics, 1-ring aromatics $\left(\mathrm{A}_{1}\right), 2^{+}$-ring aromatics $\left(\mathrm{A}_{2+}\right)$ and other oxygenates (acids and esters). Their yields on a dry basis are defined as: 
$\mathrm{Y}_{\mathrm{j} \text {,dry basis }}=\frac{\mathrm{F}_{\mathrm{j}}}{\mathrm{F}_{\text {oxygenates }}} 100$

where $F_{j}$ and $F_{\text {oxygenates }}$ refer to the hourly mass flows of each hydrocarbon lump and the oxygenates in bio-oil (after excluding water), respectively. The experimental error in the yield calculations was of $\pm 2 \mathrm{wt} \%$, as determined from experiments carried out in triplicate in a pre-experimental phase and also during the development of the present study.

The production of phenolic and aromatic compounds has also been quantified by defining a turnover number (TON) that allows relating these target products with the features of the catalyst, namely acidity, metal content and porous structure:

$\mathrm{TON}=\frac{\mathrm{W}_{\mathrm{tp}}}{\mathrm{d}_{\mathrm{H}^{+}} \mathrm{d}_{\mathrm{M}}}$

where $W_{t p}$ is the mass of the target product produced at each hour on stream, and $d_{H+}$ and $d_{M}$ are respectively defined as the density of acidic and metallic sites, expressed in units of $\mathrm{mmol}_{\mathrm{H}+} \mathrm{cm}^{-3}$ and $\mathrm{g}_{\mathrm{M} \mathrm{cm}^{-3}}$.

\section{Results and discussion}

\subsection{Catalyst characterization}

The main compositional and textural properties of the fresh zeolite-supported catalysts and the bulk FeMoP are listed in Table 1. From ICP-MS analyses, the impregnated metal amounts on each of the supported catalysts were quantified, hence verifying that the metal content is similar and allows for the comparability of the results obtained with each of them.

The BET specific surface of the FeMoP/H $\beta$ and FeMoP/HZ supported catalysts was comparable $\left(356-366 \mathrm{~m}^{2} \mathrm{~g}^{-1}\right)$, while the specific surface for the FeMoP/HY catalyst was double $\left(750 \mathrm{~m}^{2} \mathrm{~g}^{-1}\right)$. This is explained by a notably higher proportion of micropore volume $\left(0.207 \mathrm{~cm}^{3} \mathrm{~g}^{-1}\right)$ in contrast to the other two catalysts $\left(0.082-0.084 \mathrm{~cm}^{3} \mathrm{~g}^{-1}\right)$, for overall similar total pore volumes $\left(0.215-0.273 \mathrm{~cm}^{3} \mathrm{~g}^{-1}\right)$. This result was expected considering the differences among the frameworks of each zeolite. Two different types of perpendicular elliptic channels define the structure of the HZSM-5 zeolite: straight channels of $0.53 \times 0.56 \mathrm{~nm}$ and sinusoidal ones of $0.51 \times 0.55 \mathrm{~nm}$, without cages in the 
intersections. On the other hand, HBeta zeolite consists of channels of $0.56 \times 0.56 \mathrm{~nm}$ and $0.76 \times 0.64 \mathrm{~nm}$, forming openings of $0.56 \times 0.65 \mathrm{~nm}$, while the HY zeolite presents a structure with three-directional channels $0.74 \mathrm{~nm}$ wide which intersect forming cages (cavities) of a diameter of $1.24 \mathrm{~nm}$ [38].

The $\mathrm{NH}_{3}$-TPD profiles for the fresh supported catalysts (Fig. S1) and bulk FeMoP presented two main peaks: (i) a low-temperature peak corresponding to weak (w) sites on the catalyst surface, and a (ii) high-temperature peak (well-defined in FeMoP/HZ and FeMoP/HY catalysts) assigned to Lewis sites of a moderate (m) acid strength [39]. The FeMoP/HY was found to be the most acidic catalyst in terms of total acidity (1.11 mmol $_{\mathrm{NH} 3} \mathrm{~g}^{-1}$ ), followed by the FeMoP/HZ, FeMoP/H $\beta$ and the bulk FeMoP catalysts $\left(0.57,0.42\right.$ and $0.24 \mathrm{mmol}_{\mathrm{NH} 3} \mathrm{~g}^{-1}$, respectively. The total acidity tendency of the supported catalysts was observed to be inverse to that of the $\mathrm{Si} / \mathrm{Al}$ ratio of the zeolites (Table S1). Interestingly, the amount of moderately strong sites in the FeMoP/H $\beta$ catalyst is very small and the maximum of the corresponding peak is poorly defined. Additionaly, the maximum of the low-temperature peak slightly shifts to higher temperatures in the case of the supported FeMoP/HZ and FeMoP/H $\beta$ catalysts (239$\left.245^{\circ} \mathrm{C}\right)$ in contrast to the FeMoP/HY catalyst $\left(215^{\circ} \mathrm{C}\right)$. Similarly, the moderate acidity peak maxima also shift towards higher temperatures, registering temperature values of 337 and $378{ }^{\circ} \mathrm{C}$ for the $\mathrm{FeMoP} / \mathrm{HY}$ and $\mathrm{FeMoP} / \mathrm{HZ}$ catalysts, respectively. The deconvolution of the $\mathrm{NH}_{3}$-TPD curves in Fig. $\mathrm{S} 1$ was carried out to quantify the relative population of weak and moderate acidic sites, as $f_{w}$ and $f_{m}$, respectively (Table 2). Comparing the acidity of the supported catalysts with that of the bulk FeMoP, we observed a clear increase of weak acidity of the supported FeMoP/H $\beta$ catalyst, while moderate acidity was more relevant on the catalysts supported on $\mathrm{HZ}$ and $\mathrm{HY}$ zeolites.

Table 2. Composition and textural properties of the fresh catalysts

\begin{tabular}{lcccc}
\hline & FeMoP/H $\beta$ & FeMoP/HZ & FeMoP/HY & Bulk FeMoP \\
\hline $\mathrm{Fe}(\mathrm{wt} \%)^{\mathrm{a}}$ & 4.70 & 5.41 & 5.44 & 26.60 \\
$\mathrm{Mo}(\mathrm{wt} \%)^{\mathrm{a}}$ & 6.96 & 7.73 & 7.65 & 38.03 \\
$\mathrm{P}(\mathrm{wt} \%)^{\mathrm{a}}$ & 4.28 & 4.66 & 4.71 & 22.46 \\
$\mathrm{~S}_{\text {BET }}\left(\mathrm{m}^{2} \mathrm{~g}^{-1}\right)^{\mathrm{b}}$ & 366 & 356 & 750 & 6 \\
$\mathrm{~V}_{\text {pore }}\left(\mathrm{cm}^{3} \mathrm{~g}^{-1}\right)^{\mathrm{b}}$ & 0.215 & 0.273 & 0.224 & 0.079 \\
$\mathrm{~V}_{\text {micropore }}\left(\mathrm{cm}^{3} \mathrm{~g}^{-1}\right)^{\mathrm{b}}$ & 0.084 & 0.082 & 0.207 & 0.002 \\
\hline
\end{tabular}




\begin{tabular}{|c|c|c|c|c|}
\hline $\begin{array}{l}\text { Total acidity } \\
\left(\mathrm{mmol}_{\mathrm{NH} 3} \mathrm{~g}^{-1}\right)^{\mathrm{c}}\end{array}$ & 0.42 & 0.57 & 1.11 & 0.24 \\
\hline$f_{w}(\%)$ & 79 & 47 & 34 & 48 \\
\hline $\mathrm{f}_{\mathrm{m}}(\%)$ & 21 & 53 & 66 & 52 \\
\hline
\end{tabular}

${ }^{\text {a}}$ Data obtained from ICP-MS (Fe/P and Mo/P are expressed on a molar basis)

${ }^{b}$ Data obtained from $\mathrm{N}_{2}$ adsorption-desorption

cData obtained from $\mathrm{NH}_{3}$-TPD

\subsection{Evolution with time on stream of the HDO activity}

Aiming for the comparison of the activity and deactivation of the FeMoP/zeolite catalysts, the evolution with time on stream (TOS $=8 \mathrm{~h}$ ) of the distribution of the main product fractions, as described by Eq. 1 in a wet bio-oil basis, are displayed in Fig. 1. It should be mentioned that operating with a continuous bio-oil feed flow in the fixed bed reactor allows for studying the behavior of the catalysts until a pseudosteady activity state is reached, once the deposited coke amount remains constant. This way, the obtained results correspond to both the transitory period of the reaction (TOS $=0-5 \mathrm{~h}$, while progressive catalyst deactivation takes place) as well as to the pseudosteady state (TOS $>5 \mathrm{~h}$ ), which is more interesting from an industrial perspective.

In all cases, the main products at TOS $=1 \mathrm{~h}$ are gases and coke. Gases originate from decarbonylation/decarboxylation reactions and coke is formed through repolymerization/condensation reactions of bio-oil oxygenated on the outter Surface of the catalyst particles [35] and also from condesation of aromatic compounds within the micropores of the catalyst [40]. Water is also formed by dehydration of oxygenates at the initial hours of reaction for the supported catalysts and resulted the predominant product fraction for all the catalysts at TOS $>2 \mathrm{~h}$, with yields of 22-62 wt $\%$, followed by carbon products in the case of the zeolite-supported catalysts, and gas+coke products when using the bulk FeMoP phase. The fast formation of water and carbon products using the supported FeMoP catalysts evidences that the incorporation of a zeolite support hinders decarbonylation/decarboxylation reactions, favoring a higher selectivity towards carbon products, likely due to the condensation and dehydration effect that zeolites perform. On the other hand, it was observed that at TOS $>5 \mathrm{~h}$ a pseudosteady catalyst activity state is reached with all the catalysts, after which product yields remained constant. Specifically, at this pseudosteady state, the carbon product yields using the bulk FeMoP were of 16.5-17.0 wt\%, increasing up to 22.8-22.9 wt\%, 25.8- 
$26.1 \mathrm{wt} \%$ and $26.9-27.3 \mathrm{wt} \%$ (values in the TOS $=5-8 \mathrm{~h}$ range) when using the HY, H $\beta$ and HZ supports, respectively.
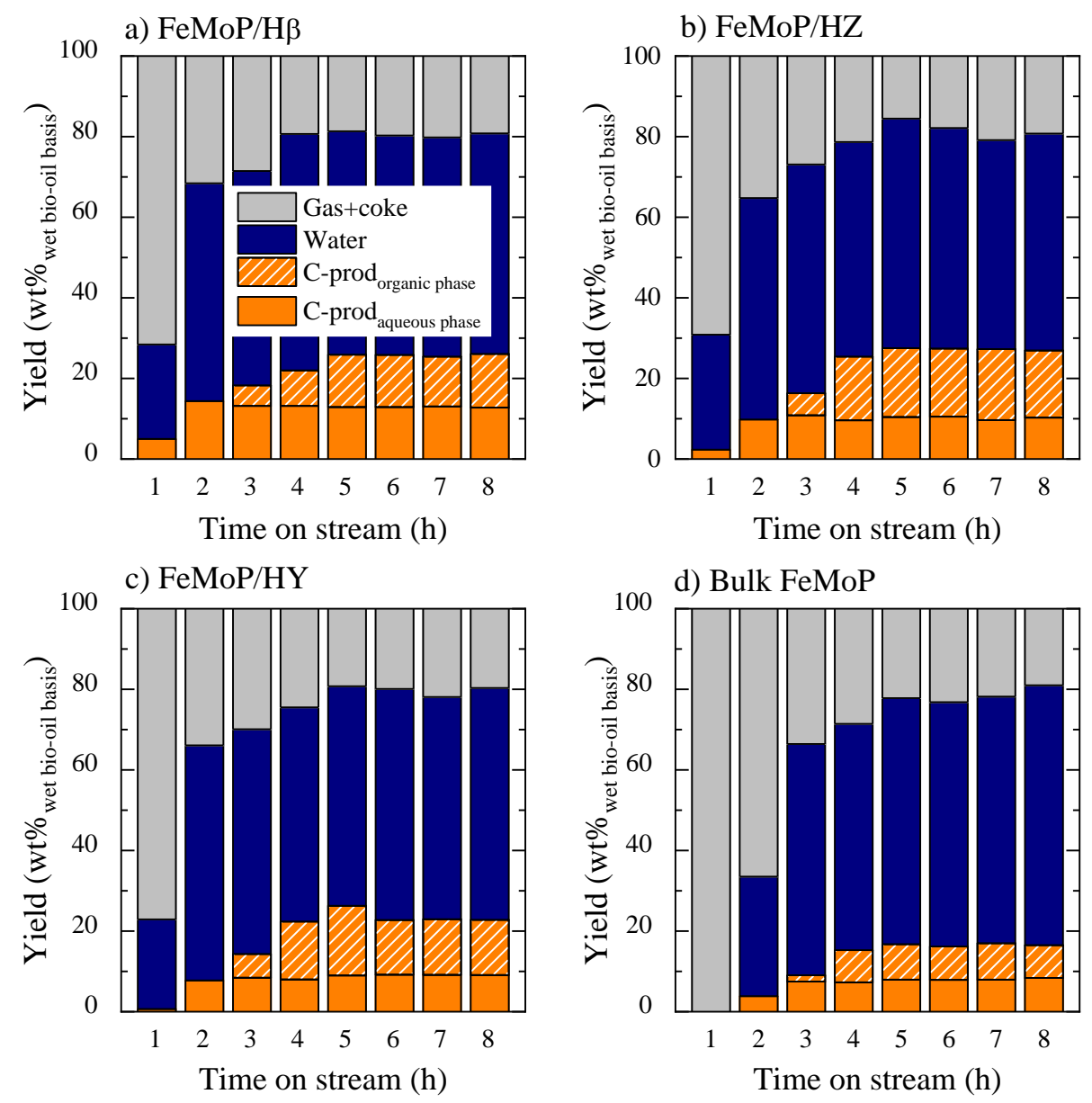

Fig. 1. Comparison of the product yields (wt\% on a wet bio-oil basis) obtained with the different catalysts.

Carbon products originate from both an aqueous and an organic product phase, with the latter developing only at TOS $>3 \mathrm{~h}$. The formation of this organic phase indicates a loss in the HDO capacity of all the catalysts due to a pronounced catalyst deactivation (by coke deposition) occurring in the initial hours of reaction [34]. An increasing trend is observed as the reaction proceeds, stabilizing at the pseudosteady activity state. In the case of the bulk FeMoP and the FeMoP/H $\beta$ catalysts, the proportion of the aqueous and organic product phases in the carbon products is similarly distributed (aqueous/organic phase ratio of $0.92-1.08$ ) while, on the other hand, the proportion of organic products is higher for the $\mathrm{FeMoP} / \mathrm{HY}$ and $\mathrm{FeMoP} / \mathrm{HZ}$ catalysts (aqueous/organic phase ratio of 1.50 and 1.63, respectively, taking average values obtained at TOS $=5-8 \mathrm{~h}$ ). This likely 
indicates that the dehydration capacity of the zeolites is closely related to the acidic features of the catalyst as well as the strength of the sites. This way, moderate acidic sites in the FeMoP/HZ and FeMoP/HY catalysts (53\% and 66\%, respectively) promote the formation of a more condensed organic phase due to a more pronounced dehydration/condensation activity in contrast to the FeMoP/H $\beta$ catalyst which presents a lower content (35\%) of these type of sites of moderate acidic strength [41].

The pseudosteady state reached by the catalyst can be explained from the consecución of a coke formation equilibrium and a balance between condensation and hydrocracking reactions of oxygenated coke precursors. A similar situation was observe don the catalytic cracking of bio-oil in FCC conditions where the coked catalyst maintained a significant remaining activity [42].

Gas composition (data not shown) was very similar in all cases, showing a prevalence of $\mathrm{CO}_{2}$ (ca. $70 \mathrm{vol} \%$ ), followed by $\mathrm{CO}$ and $\mathrm{CH}_{4}$ (ca. $15 \mathrm{vol} \%$ each). These results are in agreement with those reported in a previous work using a NiW/C catalyst, which proved to be mildly active towards HDO reactions in contrast to a noble metal-based PtPd/C catalyst. In this aforementioned work, a predominance of $\mathrm{CO}_{2}$ was also measured, as a consequence of the catalytic decomposition of acetic acid, leading to $\mathrm{CO}_{2}$ and $\mathrm{CH}_{4}$ formation. Nevertheless, the downstream gas was mainly composed by $\mathrm{H}_{2}$ as this complex hydroprocessing reaction requires working with great excess of the reactant (1000 vol\%, [43]), thus making the concentration of the produced gases negligible.

As an estimation of the evolution of the HDO activity of each catalyst [34], the amount of water contained in the aqueous product phase was measured over time on stream (Fig. 2a). Based on the fact that water is primarily formed as an end product from HDO reactions, it can be concluded that the overall HDO activity trend for the supported catalysts was equal to that of their acidity, being the FeMoP/HY catalyst the most active, and FeMoP/H $\beta$ the least. Despite the lack of a support, the amount of water measured for the bulk FeMoP was comparable to that of the FeMoP/HZ catalyst, hence confirming its noticeable HDO activity, as seen from Fig. 1d. The low water yield of the $\mathrm{FeMoP} / \mathrm{H} \beta$ catalyst is justified by its low activity as a consequence of the dilution of the bulk FeMoP in the $\mathrm{H} \beta$ zeolite support, which presents a low acidity.

According to these results, the FeMoP/HY catalyst, with an initial dehydration activity of $97.0 \%$, suffered the highest activity drop $(-11.2 \%)$, while on the other hand the 
FeMoP/H $\beta$ catalyst, with an initial activity of $82.3 \%$, only showed a slight activity decay of $-1.6 \%$ once the pseudosteady activity state was reached. Based on the analogue metal contents of all the supported catalysts (Table 2), a significantly higher dehydration activity of the FeMoP/HY catalyst is linked to both its much acidic nature (and higher proportion of moderate acidic sites) as well as to its highest specific surface, which eventually led to higher water amounts being formed in detriment to the formation of carbon products (Table 2 and Fig. 1c). The highest activity drop suffered by the FeMoP/HY catalyst can also be attributed to its framework. These precursors tend to quickly evolve towards bulkier aromatic species inside the cages of the zeolite framework, thus causing a rapid pore blockage $[38,44]$.
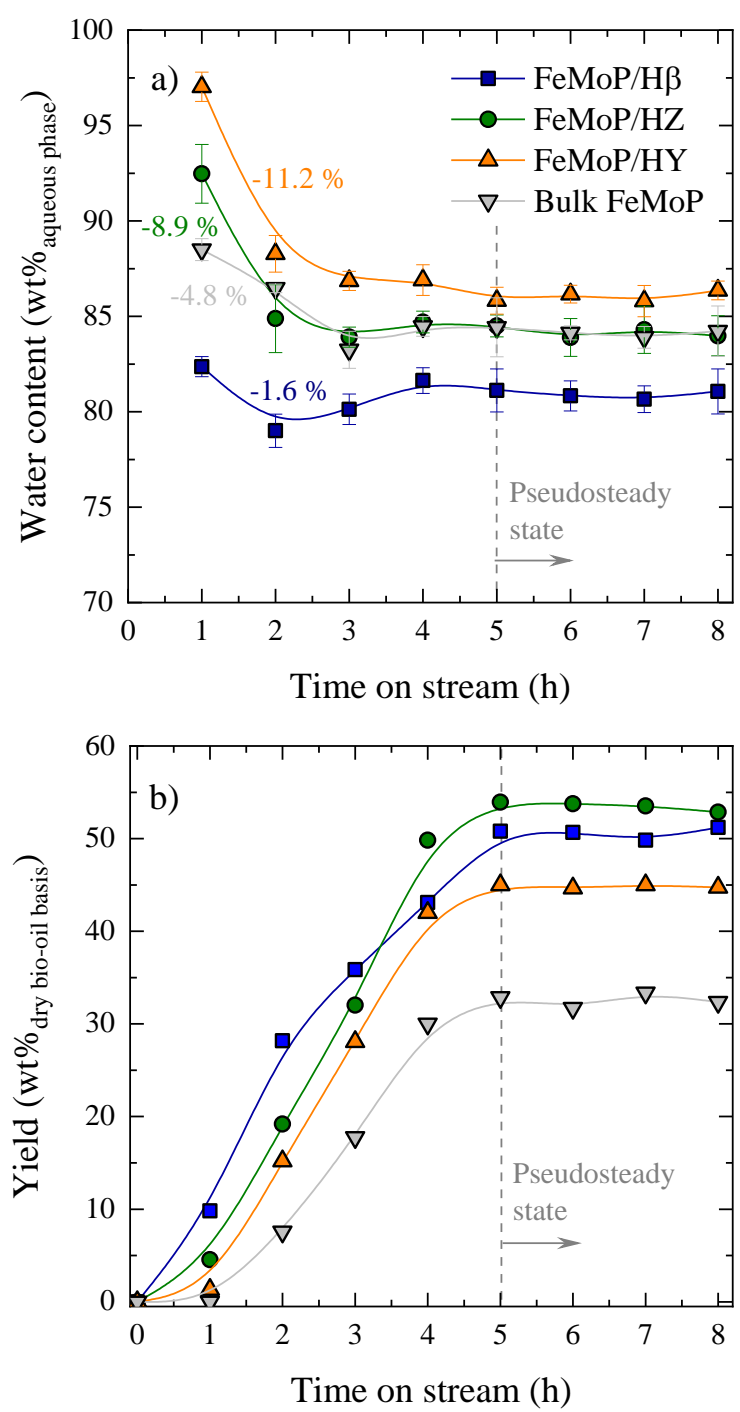

Fig. 2. Evolution with time on stream of a) the water content on the aqueous product phase for the different catalysts and b) the carbon product lumps (wt\% on a dry bio-oil 
basis) for the different catalysts

The evolution of the carbon product yields (in a dry bio-oil basis) for all the catalysts with time on stream is displayed in Fig. 2b. Comparing the results at the pseudosteady catalyst state (TOS $>5 \mathrm{~h}$ ), a significant enhancement in the production of carbon products was observed upon incorporating a zeolite support, as previously discussed from the results in Fig. 2b. Specifically, an increment from $32.6 \mathrm{wt} \%$ carbon products obtained using the bulk FeMoP phase, up to 46.5, 50.6 and $53.5 \mathrm{wt} \%$ (average pseudosteady state values for TOS $=5-8 \mathrm{~h}$ ) was achieved with the $\mathrm{HY}, \mathrm{H} \beta$ and $\mathrm{HZ}$ supports, respectively. The lowest yields obtained with the FeMoP/HY catalyst are a direct consequence of the aforementioned higher dehydration activity of the $\mathrm{Y}$ zeolite (Fig. 1c) in detriment to hydrocarbon formation [45]. Despite a higher water content being formed compared to the FeMoP/H $\beta$ catalyst (Fig. 2a), the carbon product yields obtained with the FeMoP/HZ catalyst were also higher mainly due to a higher formation of these products as derived from the organic product phase. This formation was enhanced by the moderate acidic strength of the HZ zeolite support sites (see Table 2). Interestingly, these two catalysts $(\mathrm{FeMoP} / \mathrm{H} \beta$ and $\mathrm{FeMoP} / \mathrm{HZ})$ present the more suitable shape selectivity in order to inhibit the deposition of coke, since their tridimensional channel structure (without cages in the crystalline channel intersections) favors the sweeping of coke precursors towards the reaction medium [38]. On the other hand, the remaining activity of the catalyst in the pseudosteady state is higher when the porous texture of the catalyst promotes the diffusion of coke precursors to the reaction medium. The moderate HDO activity of the metallic FeMoP function is also playing in favor of obtaining higher yields of interesting products, like phenolics and aromatics. This performance is opposed to what would occur with noble metal-based catalysts at very similar experimental conditions which provide excessive activity when these unsaturated components are targeted [34].

Regarding a relation between the target product formation and the catalyst features, a turnover number (TON) was defined on the basis of the density of acidic and metallic sites (Eq. 3). The calculations were made from the results of Table 2 and only considering phenolic and aromatic compounds as target products. Fig. 3 displays the evolution of the TON over time on stream for all the catalysts, which allows for assessing a normalized activity of the catalysts. Due to a higher density of acidic sites, 
the FeMoP/HY presented a significantly lower activity towards the targeted chemicals. On the contrary, the lower densities of acidic sites of the FeMoP/H $\beta$ and especially of the $\mathrm{FeMoP} / \mathrm{HZ}$ catalysts provided a higher activity in normalized terms, with the latter enhancing the formation of an organic product fraction, and hence providing the best results in terms of yields of aromatics and phenolics.

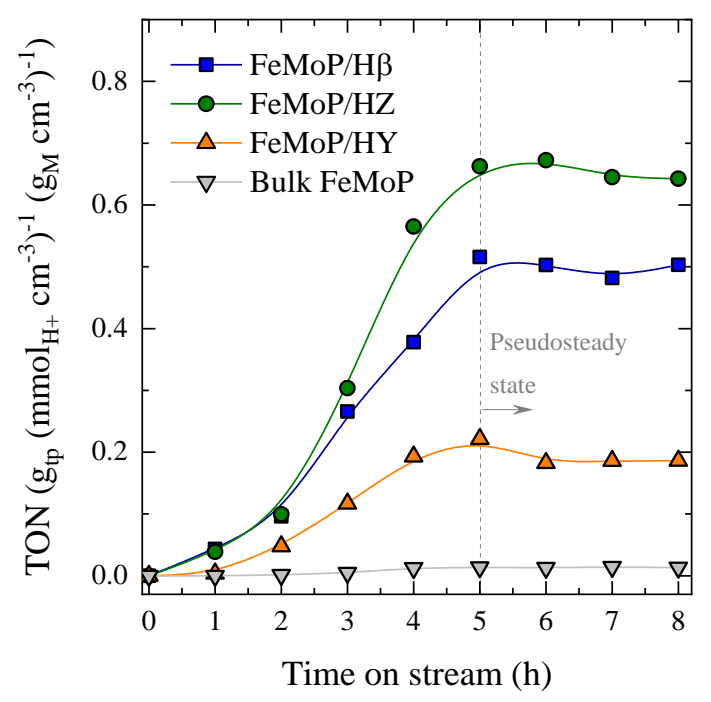

Fig. 3. Evolution with time on stream of the TON number

\subsection{Performance of the catalysts in the pseudosteady activity state}

The distribution of the yields of the carbon product lumps at TOS $=8 \mathrm{~h}$ for all the catalysts is depicted in Fig. 4. These results, corresponding to the pseudosteady activity state of the catalysts, are of great interest in order to select the most suitable catalysts for an upscaling of the process. In all cases the main lump was that of oxygenated components (comprising methanol, acetic acid and acetone), followed by total aromatics $\left(\mathrm{A}_{1}\right.$ and $\mathrm{A}_{2+}$ ), phenol + phenolics, ketones (with lower yields for the FeMP/HY and bulk FeMoP catalysts), alkanes and other oxygenates. The FeMoP/H $\beta$ catalyst showed to be the most selective towards the formation of oxygenates $(20.1 \mathrm{wt} \%)$ with the lowest HDO activity (as concluded from Fig. 2a) providing also high amounts of ketones (6.9 $\mathrm{wt} \%$ ), phenol and phenolics (5.0 $\mathrm{wt} \%$ and $5.2 \mathrm{wt} \%$, respectively) and total aromatics (11.6 wt \%). The low concentration of alkanes $(0.4 \mathrm{wt} \%)$ using the FeMoP/H $\beta$ also evidences that cracking reactions are limited, as a consequence of the low acidity of this catalyst (Table 2 ). On the contrary, the FeMoP/HZ was not only the most favorable for 
the overall formation of carbon products (see Fig. 1b and Fig. 1b), but specifically also the most selective towards aromatic compounds (13.6 wt\% total aromatics), as well as phenol (6.0 wt\%). For a more detailed quantification, Table S2 in the Supplementary Material provides the concentrations of the main chemicals of interest within the phenol/phenolics and aromatic yields, as measured by GCxGC-MS. Interestingly, while $\mathrm{A}_{1}$ and $\mathrm{A}_{2}$ lumps consist of a very wide variety of alkyl-substituted mono- and diaromatic compounds, with no clear presence of any main compound, the lump of phenolics contains high concentrations of 2- and 3-methylphenol, as well as 2,4- and 2,6-dimethylphenol, while the concentration of other phenolics was significantly lower (not included in the Table).

The suitability of the HZ zeolite for the production of phenolics and aromatics has previously been reported for the catalytic transformation of bio-oil into hydrocarbons through a 2-step thermo-catalytic approach [46,47], and it is also known for its good selectivity towards aromatics in the catalytic pyrolysis of biomass $[48,49]$. According to all these authors, the acidic sites of these zeolites promote the dehydration and aromatization reactions, however, the shape selectivity of the MFI framework plays a key role in selectivity and deactivation. It is also to mention that the utilization of solely the HZSM-5 zeolite as catalyst (with no metallic function), at the temperature operated in this study $\left(450{ }^{\circ} \mathrm{C}\right)$, would highly promote cracking reactions, with a significant formation of gas products (results not shown) and increased coke formation, which completely deactivates the catalyst within less than an hour. 


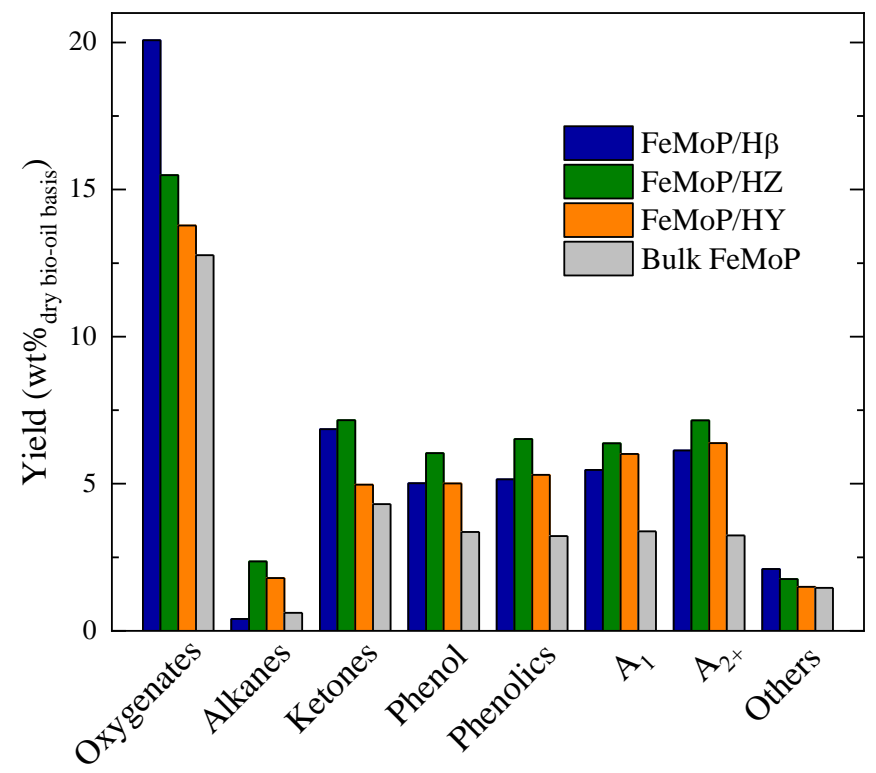

Fig. 4. Comparison of the carbon product lump yields for the different catalysts at TOS $=8 \mathrm{~h}$ (at the pseudosteady state of the catalysts).

The relative content of the three components in the lump of oxygenates (methanol, acetone, acetic acid) is similar in all cases, detecting a majority of acetic acid with all catalysts (see Fig. S2). This similarity among the results obtained with all the catalysts suggests that the selective formation of each component in the oxygenate fraction (or the interconversion among them) is dependent on the metallic function, and the support does not have significant influence. Despite not being the targeted products of interest in this work, it should also be mentioned that the aforementioned components comprised in the lump of oxygenates also present interest on their own, since they allow for being upgraded for $\mathrm{H}_{2}$ production purposes.

Despite observing significant differences in the carbon product yields obtained with the bulk FeMoP phase and the supported catalysts in Fig. 1, when assessing the concentration of the lumps of these products (see Fig. S2) the results for the bulk FeMoP and the FeMoP/H $\beta$ catalyst are analogue. This indicates that the activity in this case is predominantly determined by the metallic phase of the catalyst, while the utilization of a barely acidic zeolite support aids mainly for increasing carbon product yields due to dehydration effects, but with no effect on their composition. On the other hand, upon increasing catalyst acidity this activity is enhanced, increasing the yields of unsaturated compounds (aromatics and phenolics). The promotion of the selectivity 
towards hydrocarbons upon increasing the amount of weaker acidic sites in the catalyst has also been reported by Xing et al. [50] on the hydrodeoxygenation of eugenol, which is in agreement with the results in this study. Nonetheless, and despite detecting high concentrations of oxygenates in the products, the process was overall more selective towards the desired aromatic and phenolic components, accounting for a 42.5-50.7 wt $\%$ of the total liquid carbon products when using the zeolite-supported catalysts (Fig. S2). Interestingly, the concentration of oxygenates was the lowest for the FeMoP/HZ catalyst (29.3 wt\%).

All in all, assessing the obtained yields of the main product fractions (Fig. 1) and of the carbon products in the pseudosteady activity state (Fig. 2b), it can be concluded that a compromise among (i) a mild total catalyst acidity, (ii) a predominance of weak acidic sites and (iii) a framework structure without cages in the zeolite channel intersections, makes the $\mathrm{HZ}$ zeolite support (hence the FeMoP/HZ catalyst) the most encouraging for the conversion of raw bio-oil towards hydrocarbons. The results in this study also indicate that prospective research in raw bio-oil HDO over a FeMoP/HZ catalyst should preferably be directed towards improving catalyst performance by modifying the acidic properties of the catalyst.

\subsection{Coke deposition and catalyst deactivation}

In order to gather insights into the effect of the different zeolite supports and reaction medium composition on coke formation and nature, the characterization of the used catalysts (at TOS $=8 \mathrm{~h}$ ) was carried out. Through the obtained TG-TPO profiles, shown in Fig. 5, the existence of three different fractions was identified, namely thermal lignin (TL, peak maximum at $369^{\circ} \mathrm{C}$ ) and two types of catalytic coke (CK): a low-temperature (CK1, peak maximum at $415-449^{\circ} \mathrm{C}$ ) and a high-temperature (CK2, peak maximum at $519^{\circ} \mathrm{C}$ ) coke. While TL is known to originate from the repolymerization of the oxygenates present in bio-oil and has a tendency for depositing on the outside of the catalyst particles, $\mathrm{CK}$ is predominantly deposited over the metallic sites of the catalyst and it is formed from the oxygenates and hydrocarbons through condensation reactions catalyzed by acidic sites $[9,51,52]$. The difference between the combustion temperatures of $\mathrm{CK} 1$ and $\mathrm{CK} 2$ could be ascribed to a more external location on the metallic sites of CK1 and its lower condensation degree, which facilitates its combustion $[35,53]$. 


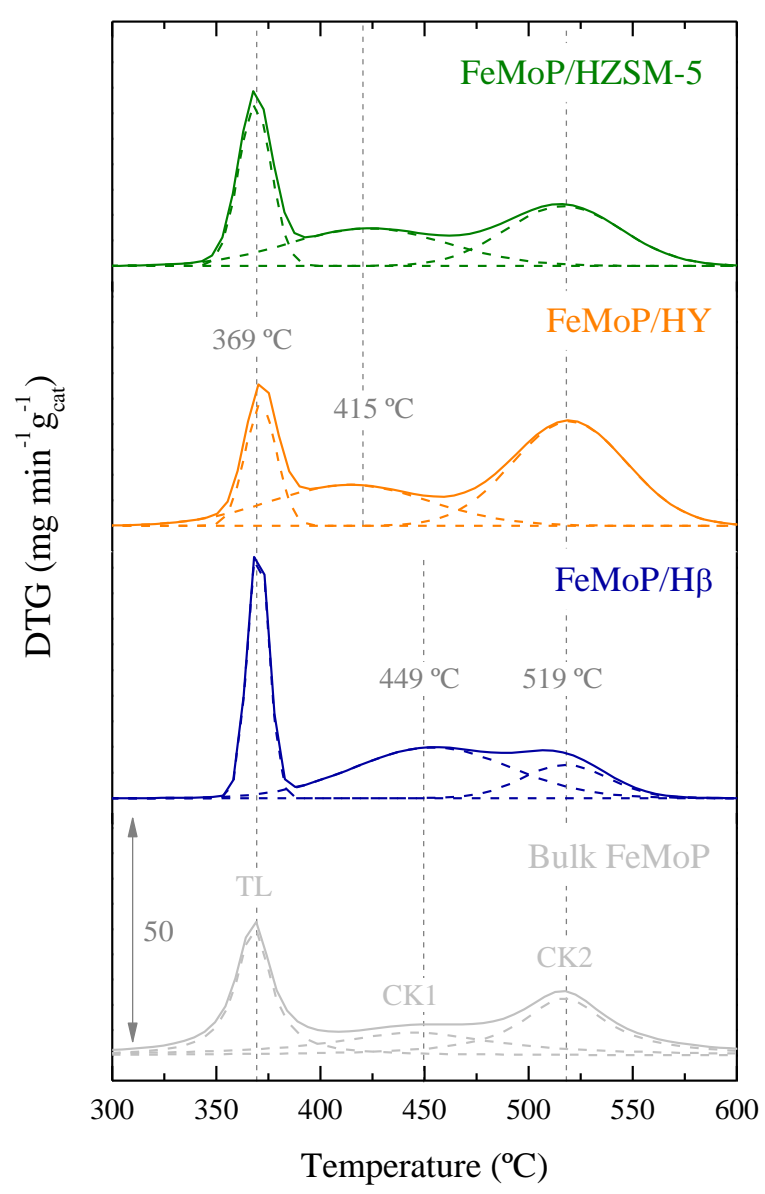

Fig. 5. TPO profiles of the used catalysts.

Interestingly, the peak maxima for TL and CK2 are fixed for all catalysts, however, there is a shift towards higher temperatures for CK1 in the case of the used bulk FeMoP and $\mathrm{FeMoP} / \mathrm{H} \beta$ catalysts $\left(449^{\circ} \mathrm{C}\right)$. This indicates that $\mathrm{CK} 1$ has a more condensed and developed nature [53], in contrast to a "lighter" one deposited on the used FeMoP/HY and $\mathrm{FeMoP} / \mathrm{HZ}$ catalysts $\left(415^{\circ} \mathrm{C}\right)$. The different mechanisms of coke deposition (thermal and catalytic) and the influence of acidity on these mechanisms likely explain these results [9]. This way, the acidic sites of the HZ and HY zeolite supports are capable of cracking some of the TL precursors, thus yielding a less developed catalytic coke with lower combustion temperatures $\left(\mathrm{CK} 1,415^{\circ} \mathrm{C}\right)$.

The total coke content as well as the relative contents of the different coke fractions were calculated from the TPO profiles in Fig. 5 using a MATLAB routine [53], and are listed in Table 3. The lowest coke content was measured for the bulk FeMoP catalyst 
(40.4 wt\%), while in the case of the supported catalysts, FeMoP/H $\beta$ showed the lowest amount of coke followed by $\mathrm{FeMoP} / \mathrm{HZ}$ and FeMoP/HY (52.5 wt\%), respectively. Interestingly, this coke content trend correlates with the drops of the overall HDO activity observed in Fig. 1 and Fig. 2a, as well as the decrease in the acidity and the channel size of the zeolite support (Table 2). Compiling these results, it was observed that a higher acidity favored the advance of water formation with a parallel decrease in the formation of carbon products, which mainly evolve towards coke, according to the results in Table 3.

Table 3. Total coke content and distribution of the different coke fractions deposited on the used catalysts

\begin{tabular}{lc|ccc}
\hline & Coke content (\%) & TL (\%) & CK1 (\%) & CK2 (\%) \\
\hline FeMoP/H $\beta$ & 44.1 & 34 & 49 & 17 \\
FeMoP/HZ & 46.0 & 29 & 33 & 38 \\
FeMoP/HY & 52.5 & 17 & 29 & 54 \\
Bulk FeMoP & 40.4 & 36 & 27 & 37 \\
\hline
\end{tabular}

Regarding the different coke fractions identified in Fig. 5, TL accounted for 29-36 wt $\%$ of the total coke, with the exception of the FeMoP/HY catalyst, in which this proportion decreased down to $17 \mathrm{wt} \%$, with a parallel increase in the proportion of CK2 fraction (54 wt\%). This greater total coke formation of the FeMoP/HY catalyst $(52.5 \mathrm{wt})$ in contrast with the other zeolite-supported catalysts is explained by the presence of cages in the microporous channel intersections of the HY zeolite, which promote the confinement of coke precursors [54]. On the other hand, the absence of these cavities in the structure of the HZSM-5 zeolite allows the sweeping of coke precursors by water in the reaction media [55].

Based on these results, catalyst acidity is one of the differentiating factors on the coke formation mechanisms. In fact, a clear dependence of catalyst acidity on both the total deposited coke and the relative contents of the different coke fractions are observed in Fig. 6. The total amount of coke increased upon increasing the catalyst acidity, which is consistent with the results reported by Marafi and Furimsky [56]. Furthermore, a lower metal sites/acidic sites ratio can explain both the increase in the amount of coke and the 
faster activity drop previously described in Fig. 2a [57]. The high acidity of the HY zeolite, as well as its framework structure, favors the formation of coke and particularly the CK2 fraction. Regarding the evolution of each fraction with the catalyst acidity, the important role of the acidic sites, specifically moderate ones (Fig. 6b), in the condensation-cracking of coke precursors can be observed. The decrease in TL is attributed to the cracking of its phenolic branches, while the drop in the proportion of CK1 fraction can be associated with coke aging and condensation towards CK2 fraction, which are favored in the acidic sites [58]. Indeed, this result shows a correlation between the amount of moderate acidic sites and the amount of CK2 fraction, which is formed by coke structures likely deposited on these sites. Hence, it can be concluded that catalyst acidity not only plays a determining role in the HDO reaction mechanism [45,59], but also in the deactivation of the catalyst. In this sense, the direct correlation between the catalyst acidity (and the strength of the acidic sites) and the formation of coke has already been established [60].

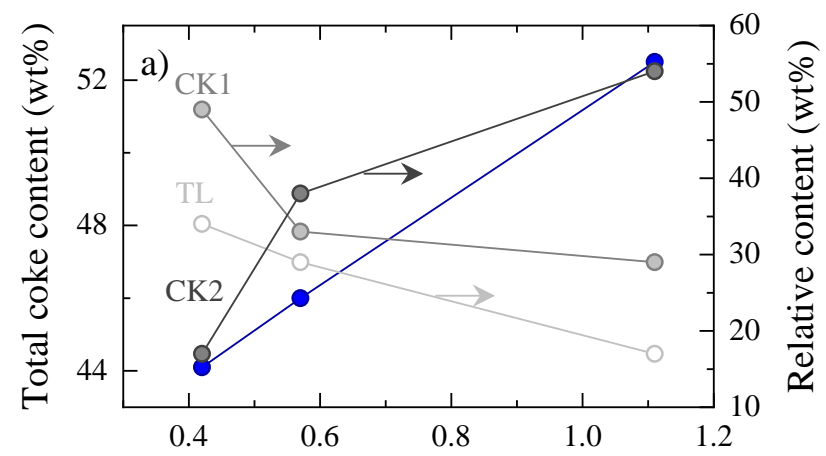

Catalyst acidity $\left(\mathrm{mmol}_{\mathrm{NH} 3} \mathrm{~g}_{\text {cat }}{ }^{-1}\right)$

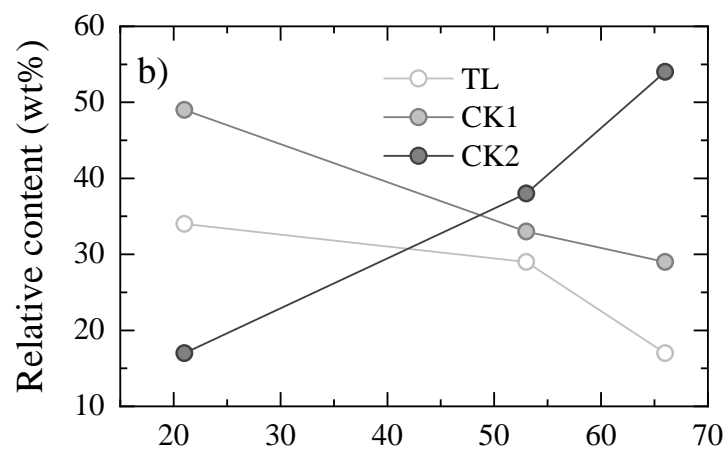

Amount of moderate sites (\%)

Fig. 6. Effect of a) catalyst acidity and b) the amount of moderate acid sites on the total coke content and relative content of the distinct coke fractions deposited on the zeolitesupported FeMoP catalysts 
The results of the previous sections point out that the most acidic catalyst (FeMoP/HY) shows the greatest advance of HDO reactions. However, considering our goal of hydrocarbon production, this catalyst leads to high amounts of water being formed, hence decreasing the yields of hydrocarbons due to favored dehydration pathways. Moreover, this catalyst is also the most active in terms of coke formation, since it promotes aromatization and condensation mechanisms by its strong acidic sites. Therefore, the use of a mildly acidic support like the $\mathrm{HZ}$ zeolite (FeMoP/HZ catalyst) is more interesting for a potential scale-up of the process, considering that its properties maximize the hydrocarbon production simultaneously limiting the catalyst deactivation by coking.

\section{Conclusions}

In this work, zeolites are reported as promising supports for FeMoP catalysts on the hydrodeoxygenation (HDO) of raw bio-oil towards aromatics and phenolics, providing a high activity, selectivity and stability than a bulk FeMoP catalyst. The key role of the acidic and structural features of the zeolites in product distribution and composition has been demonstrated, as well as their influence on coke formation and catalyst deactivation.

After a fast initial transitory deactivation period (TOS $=1-4 \mathrm{~h}$ ), at $\operatorname{TOS}=5 \mathrm{~h}$ the catalysts reach a pseudosteady activity state. In these conditions, the total acidity of the catalyst and the balance between weak and moderate acidic sites are determining for enhancing carbon product yields. In this regard, catalysts with lower acidity (namely FeMoP/H $\beta$ and FeMoP/HZ) were observed to be more suitable for attaining milder HDO conversions, limiting the formation of water. Complementarily, the presence of moderately acidic sites (35-53\%) has resulted beneficial for enhancing dehydration reactions towards the formation of an organic product phase, hence leading to higher carbon product yields. Overall, the $\mathrm{FeMoP} / \mathrm{HZ}$ is the most selective catalyst for the stable production of unsaturated compounds $(6.0 \mathrm{wt} \%$ phenol, $6.5 \mathrm{wt} \%$ phenolics and $13.6 \mathrm{wt} \%$ total aromatics yields, on a dry bio-oil basis). On the other hand, the most acidic FeMoP/HY catalyst, with the strongest acidity, provides an excessive advance of dehydration and condensation pathways, leading to higher water yields and lower carbon products formation. 
Coke formation and composition is also heavily influenced by catalyst acidity and strength. Coke formation follows an equal trend to that of the total catalyst acidity, also presenting a more condensed nature when stronger acidity becomes dominant. The weaker acidity of $\mathrm{HZ}$ and $\mathrm{H} \beta$ zeolites prevents coke precursors from evolving towards more condensed polyaromatic structures of coke. Simultaneously, their frameworks, without cages in the channel intersections, limit coke formation by sweeping coke precursors towards the reaction medium. Consequently, these catalysts have a constant remaining activity for the formation of carbon products after the initial deactivation period. On the other hand, the high acidity of HY zeolite and its more severe shape selectivity favor the trapping and condensation of coke precursors in the zeolite cages, resulting in a more pronounced deactivation.

\section{Acknowledgements}

This work was carried out with the support of the Ministry of Economy and Competitiveness of the Spanish Government, some cofounded with ERDF funds (CTQ2015-67425-R), the Basque Government (IT2018-19) and the European Commission (Horizon H2020-MSCA RISE-2018, Contract No. 823745). Dr. Idoia Hita is grateful for her postdoctoral grant awarded by the Department of Education, University and Research of the Basque Government (POS_2015_1_0035). Authors thank Dr. Enrico Catizzone from UniCAL (Cosenza, Italy) for ICP-MS measurements.

\section{References}

[1] A. Corma, S. Iborra, A. Velty, Chem. Rev. (2007) 2411-502.

[2] O.K. Lee, D.H. Seong, C.G. Lee, E.Y. Lee, J. Ind. Eng. Chem. 29 (2015) 24-31.

[3] Z. Si, X. Zhang, C. Wang, L. Ma, R. Dong, Catalysts 7(6) (2017) 169-91.

[4] E. Lazzari, T. Schena, M.C.A. Marcelo, C.T. Primaz, A.N. Silva, M.F. Ferrão, T. Bjerk, E.B. Caramão, Ind. Crops Prod. 111 (2018) 856-64.

[5] A.R.K. Gollakota, M. Reddy, M.D. Subramanyam, N. Kishore, Renew. Sustain. Energy Rev. 58 (2016) 1543-68.

[6] J. Wildschut, F.H. Mahfud, R.H. Venderbosch, H.J. Heeres, Ind. Eng. Chem. Res. 48(23) (2009) 10324-34.

[7] Z. Ma, L. Wei, W. Zhou, L. Jia, B. Hou, D. Li, Y.Q. Zhao, Fuel 92(4) (2018) 
855-60.

[8] C.A. Mullen, A.A. Boateng, Fuel 245 (2019) 360-7.

[9] T. Cordero-Lanzac, R. Palos, I. Hita, J.M. Arandes, J. Rodríguez-Mirasol, T. Cordero, J. Bilbao, P. Castaño, Appl. Catal. B Environ. 239 (2018) 513-24.

[10] I. Hita, P.J. Deuss, G. Bonura, F. Frusteri, H.J. Heeres, Fuel Process. Technol. 179 (2018) 143-53.

[11] A.N. Kay Lup, F. Abnisa, W.M.A. Wan Daud, M.K. Aroua, J. Ind. Eng. Chem. 56 (2017) 1-34.

[12] J. He, C. Zhao, J.A. Lercher, J. Catal. 309 (2014) 362-75.

[13] E.H. Lee, R.-S. Park, H. Kim, S.H. Park, S.-C. Jung, J.-K. Jeon, S.C. Kim, Y.-K. Park, J. Ind. Eng. Chem. 37 (2016) 18-21.

[14] I. Kim, A.A. Dwiatmoko, J.-W. Choi, D.J. Suh, J. Jae, J.-M. Ha, J.-K. Kim, J. Ind. Eng. Chem. 56 (2017) 74-81.

[15] V.N. Sapunov, A.A. Stepacheva, E.M. Sulman, J. Wärnå, P. Mäki-Arvela, M.G. Sulman, A.I. Sidorov, B.D. Stein, D.Y. Murzin, V.G. Matveeva, J. Ind. Eng. Chem. 46 (2017) 426-35.

[16] E.A. Roldugina, E.R. Naranov, A.L. Maximov, E.A. Karakhanov, Appl. Catal. A Gen. 553 (2018) 24-35.

[17] X. Zhang, W. Tang, Q. Zhang, Y. Li, L. Chen, Y. Xu, C. Wang, L. Ma, Fuel 215 (2018) 825-34.

[18] A.R. Ardiyanti, S.A. Khromova, R.H. Venderbosch, V.A. Yakovlev, H.J. Heeres, Appl. Catal. B Environ. 117-118 (2012) 105-17.

[19] B. Yoosuk, D. Tumnantong, P. Prasassarakich, Fuel 91(1) (2012) 246-52.

[20] X. Zhang, T. Wang, L. Ma, Q. Zhang, T. Jiang, Bioresour. Technol. 127 (2013) 306-11.

[21] X. Zhang, L. Chen, W. Kong, T. Wang, Q. Zhang, J. Long, Y. Xu, L. Ma, Energy 84 (2015) 83-90.

[22] X. Zhang, Q. Zhang, T. Wang, B. Li, Y. Xu, L. Ma, Fuel 179 (2016) 312-21. 
[23] L. Nie, P.M. De Souza, F.B. Noronha, W. An, T. Sooknoi, D.E. Resasco, J. Mol. Catal. A Chem. 388-389 (2014) 47-55.

[24] Z. Pan, R. Wang, M. Li, Y. Chu, J. Chen, J. Energy Chem. 24(1) (2015) 77-86.

[25] K. Li, R. Wang, J. Chen, Energy Fuels 25(3) (2011) 854-63.

[26] S. Echeandia, B. Pawelec, V.L. Barrio, P.L. Arias, J.F. Cambra, C. V Loricera, J.L.G. Fierro, Fuel 117 (2014) 1061-73.

[27] H. Shafaghat, P.S. Rezaei, W.M.A.W. Daud, J. Ind. Eng. Chem. 35 (2016) 26876.

[28] D.J. Rensel, J. Kim, Y. Bonita, J.C. Hicks, Appl. Catal. A Gen. 524 (2016) 8593.

[29] D.J. Rensel, S. Rouvimov, M.E. Gin, J.C. Hicks, J. Catal. 305 (2013) 256-63.

[30] D.J. Rensel, J. Kim, V. Jain, Y. Bonita, N. Rai, J.C. Hicks, Catal. Sci. Technol 7 (2017) 1857-67.

[31] V. Jain, Y. Bonita, A. Brown, A. Taconi, J.C. Hicks, N. Rai, Catal. Sci. Technol 8 (2018) 4083-96.

[32] A.R. Fernandez-Akarregi, J. Makibar, G. Lopez, M. Amutio, M. Olazar, Fuel Process. Technol. 112 (2013) 48-56.

[33] B. Scholze, D. Meier, J. Anal. Appl. Pyrolysis 60(1) (2001) 41-54.

[34] T. Cordero-Lanzac, R. Palos, J.M. Arandes, P. Castaño, J. Rodríguez-Mirasol, T. Cordero, J. Bilbao, Appl. Catal. B Environ. 203 (2017) 389-99.

[35] B. Valle, P. Castaño, M. Olazar, J. Bilbao, A.G. Gayubo, J. Catal. 285(1) (2012) 304-14.

[36] I. Hita, A. Gutiérrez, M. Olazar, J. Bilbao, J.M. Arandes, P. Castaño, Fuel 145(0) (2015) 158-69.

[37] D. van Herk, P. Castaño, M. Quaglia, M.T. Kreutzer, M. Makkee, J.A. Moulijn, Appl. Catal. A Gen. 365(1) (2009) 110-21.

[38] P. Castaño, G. Elordi, M. Olazar, A.T. Aguayo, B. Pawelec, J. Bilbao, Appl. Catal. B Environ. 104(1-2) (2011) 91-100. 
[39] A.S. Al-Dughaither, H. De Lasa, Ind. Eng. Chem. Res. 53(40) (2014) 15303-16.

[40] Y. Fan, Y. Cai, X. Li, H. Yin, J. Xia, J. Ind. Eng. Chem. 46 (2017) 139-49.

[41] X. Xu, E. Jiang, Z. Li, Y. Sun, Fuel 221 (2018) 440-6.

[42] Á. Ibarra, I. Hita, M.J. Azkoiti, J.M. Arandes, J. Bilbao, J. Ind. Eng. Chem. 78 (2019) 372-82.

[43] A.H. Zacher, D.C. Elliott, M. V Olarte, H. Wang, S.B. Jones, P.A. Meyer, Biomass Bioenergy 125 (2019) 151-68.

[44] P. Castaño, G. Elordi, M. Ibañez, M. Olazar, J. Bilbao, Catal. Sci. Technol. 2(3) (2012) 504-8.

[45] P. Kumar, S. Reddy Yenumala, S.K. Maity, D. Shee, Appl. Catal. A Gen. 471 (2014) 28-38.

[46] B. Valle, A.G. Gayubo, A. Alonso, A.T. Aguayo, J. Bilbao, Appl. Catal. B Environ. 100(1-2) (2010) 318-27.

[47] B. Valle, A.G. Gayubo, A.T. Aguayo, M. Olazar, J. Bilbao, Energy Fuels 24(3) (2010) 2060-70.

[48] H. Paysepar, K.T.V. Rao, Z. Yuan, H. Shui, C. (Charles) Xu, Appl. Catal. A Gen. 563 (2018) 154-62.

[49] G. Dai, S. Wang, Q. Zou, S. Huang, Fuel Process. Technol. 179 (2018) 319-23.

[50] J. Xing, L. Song, C. Zhang, M. Zhou, L. Yue, X. Li, Catal. Today 258 (2015) 905.

[51] M. Guisnet, P. Magnoux, Appl. Catal. A Gen. 212(1-2) (2001) 83-96.

[52] C.H. Bartholomew, Appl. Catal. A Gen. 212(1-2) (2001) 17-60.

[53] T. Cordero-Lanzac, I. Hita, A. Veloso, J.M. Arandes, J. Rodríguez-Mirasol, J. Bilbao, T. Cordero, P. Castaño, Chem. Eng. J. 327 (2017) 454-64.

[54] G. Elordi, M. Olazar, G. Lopez, P. Castaño, J. Bilbao, Appl. Catal. B Environ. 102(1-2) (2011) 224-31.

[55] M. Ibáñez, B. Valle, J. Bilbao, A.G. Gayubo, P. Castaño, Catal. Today 195(1) (2012) 106-13. 
[56] M. Marafi, E. Furimsky, Energy Fuels 31(6) (2017) 5711-50.

[57] F. Regali, L.F. Liotta, A.M. Venezia, V. Montes, M. Boutonnet, S. Järås, Catal. Today 223 (2014) 87-96.

[58] T. Cordero-Lanzac, A. Ateka, P. Pérez-Uriarte, P. Castaño, A.T. Aguayo, J. Bilbao, Ind. Eng. Chem. Res. 57(41) (2018) 13689-702.

[59] J. Xing, L. Song, C. Zhang, M. Zhou, L. Yue, X. Li, Catal. Today 258 (2015) 905.

[60] P.A. Nikulshin, V.A. Sal'nikov, E.O. Zhilkina, A.A. Pimerzin, Catal. Ind. 6(4) (2014) 338-47. 